\title{
ON THE INFINITE DIMENSIONALITY OF THE DOLBEAULT COHOMOLOGY GROUPS
}

\author{
HENRY B. LAUFER ${ }^{1}$
}

\begin{abstract}
Let $M$ be an open subset of a Stein manifold without isolated points. Let $\boldsymbol{\Omega}^{p}$ be the sheaf of germs of holomorphic $p$-forms, on $M$. Then $H^{q}\left(M, \mathbf{\Omega}^{p}\right)$ is either 0 or else infinite dimensional. $H^{q}(M, \delta)$ may be nonzero and finite dimensional if $M$ is the regular points of a Stein space or if $\delta$ is an arbitrary coherent sheaf over $M$.
\end{abstract}

Let $M$ be a complex manifold. Let $\Omega^{p}$ be the sheaf of germs of holomorphic $p$-forms over $M$. If $M$ is a Stein manifold, then $H^{q}\left(M, \Omega^{p}\right)=0$ for $q \geq 1$ [3, VIII.A, 14, Cartan's Theorem B, p. 243] while $H^{0}\left(M, \Omega^{p}\right)$ is an infinite dimensional Fréchet space, so long as $M$ does not consist of a finite number of points. If $M$ is a compact manifold, then $H^{q}\left(M, \Omega^{p}\right)$ is finite dimensional for all $p$ and all $q$ [3, VIII.A, 10, p. 245]. In this paper, we shall examine the possible dimensions for $H^{q}\left(M, \Omega^{p}\right)$, the Dolbeault cohomology groups, under the assumption that $M$ is an open subset of a Stein manifold and that $M$ does not have any isolated points. By considering the natural topology on $H^{q}\left(M, \Omega^{p}\right)$ Siu showed [7, Theorem $\left.A, p .17\right]$, under much more general assumptions, that $H^{q}\left(M, \Omega^{p}\right)$ cannot be countably infinite dimensional. The topology on $H^{q}\left(M, \Omega^{p}\right)$ is that induced from the topology of uniform convergence on compact sets for all derivatives for $C^{\infty}$ differential forms. $H^{q}\left(M, \Omega^{p}\right)$ is then a linear topological space. Let $R^{p, q}$ be the closure of 0 in $H^{q}\left(M, \Omega^{p}\right)$. Then $H^{q}\left(M, \Omega^{p}\right) / R^{p, q}$ is a separable Fréchet space. Siu essentially showed that $R^{p, q}$ cannot be countably infinite dimensional. The main result of this paper is that $H^{q}\left(M, \Omega^{p}\right) / R^{p, q}$ is either 0 or infinite dimensional. The author previously proved a special case in $[5$, Theorem $4.5, \mathrm{p}$. 431]. Some examples are given which show that some special assumptions about $M$ and about the sheaf are needed.

Let $E^{p, q}$ be the $C^{\infty}$ differential forms on $M$ of type $(p, q)$. If $f$ is a holomorphic function on $M$, then $f$ operates on $E^{p, q}$ via multiplication. We shall also denote this endomorphism of $E^{p, q}$ by $f$. Let $\lambda$ be a holomorphic vector field on $M$, i.e., a section of the dual sheaf to $\Omega^{1}$. Then $\lambda$ induces a map, also denoted by $\lambda, \lambda: E^{p, q} \rightarrow E^{p-1, q}$ given by contraction, thinking of

Received by the editors July 29, 1974.

AMS (MOS) subject classifications (1970). Primary 32C35; Secondary 32C10.

Key words and phrases. Dolbeault cohomology, sheaf cohomology, Stein manifold, linear topological space, differential form. tion.

1 Research was partially supported by a grant from the National Science Founda- 
the vector field $\lambda$ and elements of $E^{p, q}$ and $E^{p-1, q}$ as tensors. In local coordinates, if

$$
\lambda=\sum \lambda_{k}(z) \frac{\partial}{\partial z_{k}}, \quad 1 \leq k \leq n,
$$

and $\omega \in E^{p, q}$ is given by

$$
\omega=\sum \omega_{I, J}(z) d z^{I} \wedge d \bar{z}^{J}
$$

where the summation is over the multi-indices $I=\left(i_{1}, \ldots, i_{p}\right), i_{1}<\cdots<i_{p}$, and $J=\left(j_{1}, \ldots, j_{q}\right), j_{1}<\cdots<j_{q}$, then

$$
\lambda(\omega)=\sum(-1)^{\epsilon+1} \lambda_{k}(z) \omega_{I, J}(z) d z^{I^{\prime}} \wedge d \bar{z}^{J}
$$

where the summation is over $1 \leq k \leq n, J=\left(j_{1}, \ldots, j_{q}\right)$ and $I=\left(i_{1}, \ldots, i_{p}\right)$ such that $k \in I$. $I^{\prime}$ is obtained from $I$ by deleting $k_{\text {. }} \epsilon$ is given by $I \ni k$, $k=i_{\epsilon}$.

The map $\lambda$ commutes with the map $f$.

Let $D: E^{p, q} \rightarrow E^{p, q}$ be given by $D=\partial \circ \lambda+\lambda \circ \partial$. The following lemma is true for any complex manifold. $\partial f$ is a holomorphic 1 -form and $\lambda(\partial f)$ is the function obtained by the usual operation of a vector field $\lambda$ on a function $f$.

Lemma. Let $D=\partial \circ \lambda+\lambda \circ \partial$. Then $D \circ f-f \circ D=\lambda(\partial f)$.

Proof. Let $\omega \in E^{p, q}$.

$$
\begin{gathered}
(\partial \circ \lambda \circ f)(\omega)=(\partial \circ f \circ \lambda)(\omega)=\partial f \wedge \lambda(\omega)+f \wedge(\partial \circ \lambda)(\omega) \\
=\partial f \wedge \lambda(\omega)+(f \circ \partial \circ \lambda)(\omega) . \\
(\lambda \circ \partial \circ f)(\omega)=\lambda(\partial f \wedge \omega+f \wedge \partial \omega)=\lambda(\partial f \wedge \omega)+(f \circ \lambda \circ \partial)_{\omega} .
\end{gathered}
$$

From (1) and (2),

$$
(D \circ f-f \circ D)(\omega)=\partial f \wedge \lambda(\omega)+\lambda(\partial f \wedge \omega) .
$$

The right side of (3) is $\hat{C}$-linear in $\lambda$ and $C^{\infty}$-linear in $\omega$. So to complete the verification of the Lemma, it suffices to evaluate the right side of (3) in local coordinates with $\lambda=\partial / \partial z_{1}$ and $\omega=d z^{I} \wedge d \bar{z}^{J}$. There are two cases, $1 \notin I$ and $1=i_{1} \in I=\left(i_{1}, \ldots, i_{p}\right)$.

For $1 \notin I: \lambda(\omega)=0$ and $\lambda(\partial f \wedge \omega)=\partial f / \partial z_{1} \cdot \omega$, as needed.

For $1 \in I: \lambda(\omega)=d z^{I^{\prime}} \wedge d \bar{z}^{J}$ where $I^{\prime}=\left(i_{2}, \ldots, i_{p}\right)$.

$$
\begin{aligned}
\partial f \wedge \lambda(\omega) & =\sum \frac{\partial f}{\partial z_{k}} d z^{k} \wedge d z^{I^{\prime}} \wedge d \bar{z}^{J}, \\
\partial f \wedge \omega & k \notin I^{\prime}, \\
\partial \frac{\partial f}{\partial z_{k}} d z^{k} \wedge d z^{I} \wedge d \bar{z}^{J}, & k \notin I .
\end{aligned}
$$




$$
\lambda(\partial f \wedge \omega)=\sum(-1) \frac{\partial f}{\partial z_{k}} d z^{k} \wedge d z^{I^{\prime}} \wedge d \bar{z}^{J}, \quad k \notin I .
$$

$I-I^{\prime}=\{1\}$ so the summations in (4) and (5) differ only by one term, $k=1$. This completes the proof of the Lemma.

Theorem. Let $M$ be an open subset of a Stein manifold having no isolated points. Then, for any $p$ and $q, H^{q}\left(M, \Omega^{p}\right)$ is either 0 or else infinite dimensional. Let $R^{p, q}$ be the closure of 0 in $H^{q}\left(M, \Omega^{p}\right)$. Then $H^{q}\left(M, \Omega^{p}\right) / R^{p, q}$ is either 0 or else infinite dimensional.

Proof. The proof for $H^{q}\left(M, \Omega^{p}\right)$ is exactly like that for $H^{q}\left(M, \Omega^{p}\right) / R^{p, q}$, leaving out topological considerations; therefore we omit it.

For the sake of notational simplicity, let $H=H^{q}\left(M, \Omega^{p}\right) / R^{p, q}$. We shall show that if $H$ is finite dimensional, then $H=0$. For $f$ holomorphic on $M$, the action of $f$ on $E^{p, q}$ is continuous and commutes with $\bar{\partial}$. Thus, $f$ induces a continuous endomorphism on $H^{q}\left(M, \Omega^{p}\right)$ and on $H$.

Without loss of generality, we may assume that $M$ is a subset of a connected Stein manifold $S$ of dimension $n>0$. Let $I$ be the set of holomorphic functions on $S$ which, after restriction to $M$, induce the zero map of $H$ to itself. It suffices to show that $1 \in I$. Let $z_{1}, \ldots, z_{2 n+1}$ be holomorphic functions on $S$ which separate points [3, Theorem VII.C.10, p. 224]. Each $z_{i}$ acts on $H$ and so has a minimal polynomial $p_{i}\left(z_{i}\right)$, under the finite dimensional assumption on $H \cdot p_{i}\left(z_{i}\right) \in I \cdot p_{i}\left(z_{i}\right)$ has only a finite number of roots in $z_{i}$. So the common zeros on $S$ for $p_{1}\left(z_{1}\right) \ldots, p_{2 n+1}\left(z_{2 n+1}\right)$ consist of only a finite number of points, say $P_{1}, \ldots, P_{N^{\prime}}$ in $S$.

$\partial: E^{p, q} \rightarrow E^{p+1, q}$ is continuous and anticommutes with $\bar{\partial}$. If $\lambda$ is a holomorphic vector field on $S$, then $\lambda: E^{p, q} \rightarrow E^{p-1, q}$ is continuous and commutes with $\bar{\partial}$. So $\lambda \circ \partial$ and $\partial \circ \lambda$ both induce endomorphisms of $H$. The Lemma also holds for the induced maps on $H$. Thus, if $f \in I$, then also $\lambda(\partial f) \in I$. Consider a $P_{j} \in S$ from above. Let $f \in I$ have a zero at $P_{j}$ of minimal total order. $f \not 0$ since $p_{1}\left(z_{1}\right) \in I$. We claim that $f\left(P_{j}\right) \neq 0$, for suppose otherwise. Since $S$ is a Stein manifold of positive dimension, by an application of Cartan's Theorem $\mathrm{B}$, we can specify the tangent vector at $P_{j}$ for a vector field $\lambda$ on $S$. For a suitable choice for $\lambda, \lambda(\partial f)$ will have a zero

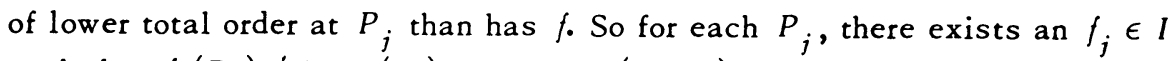
such that $f_{j}\left(P_{j}\right) \neq 0 . p_{1}\left(z_{1}\right), \ldots, p_{2 n+1}\left(z_{2 n+1}\right), f_{1}, \ldots, f_{N}$ are then elements of $I$ with no common zeroes. By [3, Corollary VIII.A.16, p. 244] there exist holomorphic functions $\left\{g_{k}\right\}$ on $S$ such that $\Sigma g_{i} p_{i}\left(z_{i}\right)+\Sigma g_{j} f_{j} \equiv 1$. Thus $1 \in I$ and $H=0$, as desired.

The Theorem does not hold under the weaker assumption that $M$ is an open subset of a Stein space, even if $M$ itself is a manifold. Consider, for example, a Riemann surface $R$ of genus at least 1 embedded as the 0 -section 
of a negative vector bundle $V$ of rank 4. See [2]. $V$ can be taken to be the direct sum of 4 line bundles, each of negative Chern class. Let $\mathcal{O}=\Omega^{0}$ be the sheaf of germs of holomorphic functions. Then $H^{1}(V, \mathcal{O}) \neq 0$ since $H^{1}(V, \mathcal{O})$ may be expanded in a power series along the fibers $[2, \mathrm{pp} .343-$ 344]. Also, $H^{1}(V, \mathcal{O})$ is finite dimensional since $V$ is strictly pseudoconvex [1, Theorem 11, p. 239]. Let $M=V-R$. Then the restriction map induces an isomorphism $H^{1}(V, \mathcal{O}) \approx H^{1}(M, \mathcal{O})$ by [6, Corollary, p. 351]. Thus $H^{1}(M, \mathcal{O})$ is nonzero and finite dimensional. By blowing down $R$ to a point $p$, we obtain a Stein space $S$ with $M$ the complement of the singular point $p$. This example also shows that $\Omega^{p}$ in the Theorem cannot be replaced by an arbitrary coherent sheaf. Namely, near $p, S$ may be embedded as a subvariety $X$ of a polydisc $\Delta$.

$$
H^{1}(S-p, \Theta) \approx H^{1}(X-p, \Theta) \text { by }\left[4 \text {, Theorem 2.2, p. 105]. } X^{\Theta}\right. \text { is a co- }
$$
herent sheaf on $\Delta$, but $H^{1}\left(\Delta-p, X^{\Theta}\right)$ is nonzero and finite dimensional.

\section{BIBLIOGR APHY}

1. A. Andreotti and H. Grauert, Théorèmes de finitude pour la cohomologie des espaces complexes, Bull. Soc. Math. France 90 (1962), 193-259. MR 27 \#343.

2. H. Grauert, Über Modifikationen und exzeptionelle analytische Mengen, Maih. Ann. 146 (1962), 331-368. MR 25 \#583.

3. R. C. Gunning and H. Rossi, Analytic functions of several complex variables, Prentice-Hall Ser. in Modern Analysis, Prentice-Hall, Englewood Cliffs, N.J., 1965. MR 31 \#4927.

4. H. B. Laufer, On sheaf cohomology and envelopes of holomorphy, Ann. of Math. (2) 84 (1966), 102-118. MR 35 \#417.

5. - On Serre duality and envelopes of holomorphy, Trans. Amer. Math. Soc. 128 (1967), 414-436. MR 36 \#5393.

6. G. Scheja, Riemannsche Hebbarkeitssätze für Cohomologieklassen, Math. Ann. 144 (1961), 345-360. MR 26 \#6437.

7. Y.-T. Siu, Non-countable dimensions of cohomology groups of analytic sheaves and domains of holomorphy, Math. Z. 102 (1967), 17-29. MR 36 \#5394.

DEPARTMENT OF MATHEMATICS, STATE UNIVERSITY OF NEW YORK, STONY BROOK, NEW YORK 11794 